

\title{
STANDARDIZATION OF SURGICAL MATERIAL KITS FOR SPINE TREATMENTS USING THE LEAN HEALTHCARE APPROACH
}

\author{
PADRONIZAÇÃO DE KITS DE MATERIAL CIRÚRGICO PARA TRATAMENTOS DE \\ COLUNA UTILIZANDO A ABORDAGEM LEAN HEALTHCARE \\ $A P P R O A C H$
}

\begin{abstract}
(iD) Luana Coelho de Morais ${ }^{1}$
(iD) Stella Jacyszyn Bachega ${ }^{2}$

(D) Naiara Faiad Sebba Calife ${ }^{3}$

iD Dalton Matsuo Tavares ${ }^{4}$
\end{abstract}

Resumo: Os kits contendo material cirúrgico selecionado podem reduzir atrasos, custos, erros e proporcionar segurança para a equipe médica e para o paciente. O objetivo da pesquisa era dimensionar os pacotes necessários para dois tipos de tratamento da coluna usando ferramentas do Lean Healthcare como suporte. O ciclo PDCA foi usado como um método de solução de problemas. Na etapa 'Plan', avaliou-se a situação atual e a necessidade de mudança por meio do Mapeamento do Fluxo de Valor, em relação à situação atual e futura. Na etapa 'Do', foi desenvolvido um plano de melhoria das mudanças, com o auxílio da abordagem 5S. Na etapa 'Check' foram utilizadas as ferramentas Kaizen, Trabalho Padronizado e Gestão Visual, para propor a implementação das mudanças. Na etapa 'Act', foi sugerido o acompanhamento das ações. Isso elimina as atividades que não agregam valor à visão do paciente ou do cliente do processo.

Palavras-chave: Lean Healthcare. Kit Cirúrgico. Produção Enxuta.

Abstract: Packs containing selected surgical material can reduce delays, costs, errors and provide safety for the medical team and for the patient. The research goal was to scale packs required for two types of spine treatment using tools from Lean Healthcare as support. PDCA cycle was used as a method of troubleshooting. In the 'Plan' stage, we evaluated the current situation and the need for change using Value Stream Mapping, regarding the current and future situations. In the "Do" stage, a plan was developed to improve the changes, with the assistance of the 5S approach. Kaizen, Standardized Work and Visual Management tools were used in the "Check" stage, to propose the implementation of the changes. In the "Act" stage, it was suggested to follow-up the actions. This eliminates the activities that do not add value in the patient's vision, or the process client.

Keywords: Lean Healthcare. Surgical Pack. Lean Production 


\section{Introduction}

Concepts and techniques used in manufacturing have helped the hospital management in the search for a more productive work, for a great environment for the professionals, in the administration of responsibilities and in the patient care process improvement. Among the approaches used, lean production has stood out in the health sector, since it is flexible and adaptable to several processes (Worth et al., 2012). According to Rutledge, Xu, \& Simpson (2015), some of the principles of lean production have been applied in hospitals, clinical laboratories and other health agencies. When applied to healthcare systems, this approach is called Lean Healthcare.

Lean Healthcare is an alternative to assist health management, showing the challenges in implementation and the benefits of it (e.g. Amran, Januddi, Nuraina, Ikbar, \& Khairanum, 2020, Tortorella, Dun, \& Almeida, 2020, Machado \& Leitner, 2010, Curatolo, Lamouri, Huet, \& Rieutord, 2013, Rutledge, Xu, \& Simpson, 2015, Giraldo, Mothersell, \& Motwani, 2015, Dobrzykowski, McFadden, \& Vonderembse, 2016, Henrique, Rentes, \& Godinho, 2016, Adebanjo, Laosirihongthong, \& Samaranayake, 2016). Costa \& Godinho (2016) do a review, classification and analysis of the literature on the subject. Graban (2011) defines lean as a strategic approach that allows hospitals to improve quality of care and reduce errors and delays, involving staff throughout the improvement process.

In the context of health, reducing delays and errors is not only desirable but essential. In the case of the Surgical Center (SC) and the Sterile Materials Center (SMC), precision and speed are vital. Surgical safety is a worldwide concern, since at least half of the incidents that occurred in the provision of health care occur in surgical procedures according to the World Health Organization (WHO). Moreover, it is estimated that half of these incidents could be prevented (Ministério da Saúde, 2015).

Thus, the use of surgical kits containing sterilized materials and selected instruments according to the medical specialty and the surgical nature can reduce the time spent by the surgical instrumentator to separate the instruments, the material costs due to the greater control of inventory, the costs with resterilization of unused materials, potential instrumentation errors, and provide safety for the physician, his or her staff and, above all, the patient.

According to Alves et al. (2016), the maladjusted composition of standard surgical packs and the non-optimized grouping of instrumental surgical packs that make up each kit according to the surgical specialty, bring consequences for the hospital, professionals and patients, referred to as operational failures such as: excessive transport; lack of instrumentality for other teams and postponement of risky surgeries; premature and excessive wear of materials by decontamination / sterilization; difficulty in systematizing the assistance provided with interruptions and constant requests for materials; delays in surgery; physical and emotional fatigue of the professionals involved; increased team irritability / tension due to failures. 
Considering the positive results of applying Lean Healthcare in several areas of the health sector, we have the following research question: how to size surgical kits for spinal treatments considering the performance of neurosurgeons and orthopedists in performing these types of procedures? Therefore, the overall goal of the research is to scale instrumental kits needed for these two types of spine treatments using Lean Healthcare tools as support. It was decided to analyze procedures for spine treatments, which are of high complexity instead of simple procedures or general surgeries, which are widely addressed in the literature. In this sense, two procedures indicated for the treatment of spinal pain were chosen as the focus of this work: Percutaneous Facet Rhizotomy and Spinal Stimulation Therapy or Spinal Neurostimulation.

Considering the importance of the Lean Healthcare approach, the advantages of using surgical kits, the need for and mandatory patient safety protocols, and the fact that Waldhausen, Avansino, Libby, \& Sawin (2010), Johnson, Smith, \& Mastro (2012), Collar et al. (2012) and Lunardini et al. (2014), have performed research in the field of Lean Healthcare implementation in surgical environments, this research is justified by the need to reduce the preparation time of surgical material and costs with instrument resterilization by means of Lean Healthcare tools. It is also noted that authors such as Byrnes, Schmitt, Tommaso, \& Occhino (2017), Cichos et al. (2019), Crosby, Lortie, Rotenberg, \& Sowerby (2019), and Marchand, Taylor, Salem, Mont, \& Marchand (2020), among others, proved in practice the benefits of surgical tray optimization, such as quality assurance and patient safety.

Kaizen, VFM, 5S, work standardization, physical process redesign and Kanban were the tools used in the study of Johson et al. (2012), which resulted in cost and overtime reduction, improvement in financial performance and planning, among others. Besides these tools, Waldhausen et al. (2010) used the workload balance to increase the satisfaction of the technical staff and patients with a significant reduction in the pre-surgical mean waiting time and a $20 \%$ increase in the number of patients operated in four hours of work.

Collar et al. (2012) presented the application of the 5 Whys tools and process redesign in a US otorhinolaryngological study, which resulted in cost reduction, increased employee satisfaction and possible increase in capacity.

Instruments for spine surgeries were the object of study for the application of lean in the work of Lunardini et al. (2014). Using process mapping and team approach to problem solving, the study resulted in the reduction of $41 \%$ of the instruments used and the consolidation of a set of instruments, generating a projected reduction of 41 thousand dollars in annual costs.

To achieve the proposed goal, this paper is organized as follows: the next section will introduce the literature review addressing current work on Lean Healthcare; in section three we present the materials and methods; the sections from four to seven will address the results and discussions according to the phases of the PDCA cycle and section eight will present the conclusion. 


\section{Literature Review}

\subsection{Lean Healthcare}

Over the years, authors have dedicated themselves to the study of Lean Healthcare in several aspects. Table 1 brings some of these, with their respective publication year and aims.

Table 1

Studies on Lean Healthcare

\begin{tabular}{|c|c|}
\hline Author & Aim of the study \\
\hline Joosten, Bongers \& Janssen (2009) & $\begin{array}{c}\text { To present an overview of lean thinking and its application to } \\
\text { health care. }\end{array}$ \\
\hline Drotz \& Poksinska (2014) & $\begin{array}{l}\text { To contribute toward a deeper understanding of the new } \\
\text { roles, responsibilities and job characteristics of employees in } \\
\text { Lean Healthcare organizations. }\end{array}$ \\
\hline $\begin{array}{l}\text { D’Andreamatteo, lanni, Lega, \& } \\
\text { Sargiacomo (2015) }\end{array}$ & $\begin{array}{l}\text { To present a comprehensive overview of the main issues } \\
\text { highlighted by research on implementation of Lean in a } \\
\text { complex contest such as the healthcare one. }\end{array}$ \\
\hline Hicks, McGovern, \& Smith (2015) & $\begin{array}{l}\text { To evaluate the application of the Lean 3P (production, } \\
\text { preparation, process) participative design method as part of a } \\
\text { pilot project to design a new endoscopy unit at Gateshead } \\
\text { Health NHS Foundation Trust. }\end{array}$ \\
\hline Sugianto et al. (2015) & $\begin{array}{l}\text { To implement Lean principles to accommodate expanding } \\
\text { volumes of gastrointestinal biopsies and to improve overall } \\
\text { laboratory processes. }\end{array}$ \\
\hline
\end{tabular}

Costa, Godinho, Rentes, Bertani, \& Mardegan (2015)

To evaluate how the five sectors of two Brazilian hospitals have implemented Lean Healthcare concepts in their operations.

Holden, Eriksson, Andreasson, Williamsson, \& Dellve (2015)

Poksinska, Fialkowska-Filipek, \& Engström (2016)

Montella et al. (2016)

Tay (2016)

Moraros, Lemstra, \& Nwankwo (2016)
To address how hospital workers' perceptions of lean varied across the role of context in shaping lean and its consequences and also, on how healthcare workers perceive lean in three Swedish hospitals.

To investigate how primary-care centers working with Lean define and improve value from the patient's perspective, and how the application of Lean Healthcare influences patient satisfaction.

To report on the application of the Lean Six Sigma (LSS) methodology to reduce the number of patients affected by sentinel bacterial infections who are at risk of HAl.

To understand how some lean improvement projects in organizations have been able to systematically focus on flow efficiency, while others tend to focus on resource efficiency.

To independently assess the effect of Lean or Lean interventions on worker and patient satisfaction, health and process outcomes, and financial costs. 


\begin{tabular}{|c|c|}
\hline Author & Aim of the study \\
\hline $\begin{array}{l}\text { Van Rossum, Aij, Simons, Eng, \& Have } \\
\qquad(2016)\end{array}$ & $\begin{array}{c}\text { To increase scientific knowledge regarding factors that diminish } \\
\text { the implementation gap and make the transition from the } \\
\text { "toolbox lean" toward an actual transformation to Lean } \\
\text { Healthcare. }\end{array}$ \\
\hline $\begin{array}{c}\text { Improta, Cesarelli, Montuori, Santillo, } \\
\text { \& Triassi (2017) }\end{array}$ & $\begin{array}{l}\text { To adopt the Lean Six Sigma to reduce the risk of healthcare- } \\
\text { associated infections (HAls), a critical quality parameter in the } \\
\text { healthcare sector. }\end{array}$ \\
\hline Tay, Singh, Bhakoo, \& Al-Balushi (2017) & $\begin{array}{l}\text { To investigate the factors that give rise to either form of } \\
\text { efficiency ('resource efficiency' or 'flow efficiency'). }\end{array}$ \\
\hline Lindskog, Hemphälä, \& Eriksson (2017) & $\begin{array}{l}\text { To expand upon lean tools and innovation enabling job } \\
\text { resources. }\end{array}$ \\
\hline $\begin{array}{l}\text { Boronat, Budia, Broseta, Ruiz-Cerdá, \& } \\
\text { Vivas-Consuelo (2018) }\end{array}$ & $\begin{array}{l}\text { To describe the application of the Lean methodology as a } \\
\text { method for continuously improving the efficiency of a urology } \\
\text { department in a tertiary hospital. }\end{array}$ \\
\hline Schonberger (2018) & $\begin{array}{l}\text { To illustrate that approach to lean by drawing from a case } \\
\text { study, in which widely scattered heart attack patients were } \\
\text { transported to a central treatment hospital in a system-wide, } \\
\text { highly coordinated program of quick response. }\end{array}$ \\
\hline Amran et al. (2020) & $\begin{array}{l}\text { To identify the factors that lead to barriers in implementing } \\
\text { lean in healthcare. }\end{array}$ \\
\hline Tortorella, Dun, \& Almeida (2020) & $\begin{array}{c}\text { To verify leadership behaviors related with lean healthcare } \\
\text { implementation and how they progress throughout the change } \\
\text { process. }\end{array}$ \\
\hline
\end{tabular}

Source: Organized by the authors.

Joosten, Bongers \& Janssen (2009) state that Lean Thinking has the potential to improve health care provision, but it should not happen superficially, without a robust methodology. It will fail, increasing existing resistance and making it more difficult to improve long-term health care.

D'Andreamatteo et al. (2015) and Moraros et al. (2016) conducted theoretical analyzes of articles that deal with the subject of Lean Healthcare. D'Andreamatteo et al. (2015) realized that Lean Healthcare is seen as a means to increase productivity and is applied mostly in the reality of hospitals. They also detected that the United States has excelled in this theme and the identified research mainly deal with challenges and factors of success and to a lesser extent, on the applicability of the methodology throughout the system. Therefore, they concluded that scholars should further explore the strengths and weaknesses of Lean Healthcare.

Moraros et al. (2016) found that Lean interventions do not statistically show any association with patient satisfaction and health outcomes. In addition, they show a negative association with 
financial costs and worker satisfaction. However, they point to potential benefits in process outcomes, such as patient flow and safety. Leaning to the conclusion that the evidence does not support the theory that Lean Healthcare leads to the improvement of quality in health.

In a follow-up to a different research using case studies, Poksinska et al. (2016) also revealed that Lean Healthcare implementations focus primarily on efficiency. Little attention is given to the patient's perspective. The authors concluded that healthcare organizations need to pay more attention to integrating the patient perspective into the application of Lean Healthcare. Value needs to be defined and value streams need to be improved based on the knowledge and clinical experience of caregivers and the preferences and needs of patients.

Drotz \& Poksinska (2014), Holden et al. (2015) and Van Rossum et al. (2016) examined the relationship between Lean Healthcare deployment and health services employee behavior. From these studies, it can be noticed that perceptions vary according to the hospital context, whereas nurses report more favorable perceptions than physicians. In general, teamwork and the decentralization of authority from the reorientation of the flow have produced positive results in the work environment with leadership flexibility, team development and everyone's involvement. Potter et al. (2005 apud Zadeh et al., 2012) found several work patterns that increased the complexity of nurses' work, including disjointed supply sources, absent supplies, repetitive trips and interruptions.

Boronat et al. (2018) and Schonberger (2018) showed the practical application of the Lean methodology in the field of health confirming some aspects of these researches and contradicting others. Boronat et al. (2018) implemented this methodology in an urology department of a tertiary hospital to enhance efficiency, obtaining significant and continuous improvements in all indicators, as well as the professional's satisfaction. Schonberger (2018), on the other hand, presents a case study in which heart attack patients are treated in a highly coordinated system-wide rapid response program by Lean Healthcare, where the results have high rates of life saving and overall lean health. The author's main conclusion is that Lean Healthcare, when practiced in this way, becomes valuable as a strategic management company methodology.

Other authors presented the use of Lean-specific tools in health and its results. Hicks, McGovern \& Smith (2015) applied the $3 \mathrm{P}$ tool (production, preparation, process) to manage medicine flows. Sugianto et al. (2015) used value-flow mapping (VFM) in a clinical laboratory and achieved a 29\% reduction in sample processing time along with continuous improvement and waste disposal. Costa et al. (2015) applied several tools, especially the VFM and DMAIC (Define, Measure, Analyze, Improve and Control) in hospitals obtaining improvements in financial aspects, productivity and capacity, and lead time reduction. Lindskog et al. (2017) also used the VFM together with the 5S, since they argue that these lean tools are considered work related resources for innovation in the initial phase of lean implementation. 
Costa et al. (2015) and Improta et al. (2017) used Lean Six Sigma as a tool to identify variables that affect the risk of healthcare-associated infections (HAls) and to implement corrective actions to improve the process of care performance. The former resulted in a $20 \%$ reduction in the mean number of days of hospitalization between the pre-intervention and the control phases, and a decrease in the average number of hospitalization days. The latter had a reduction in the number of patients colonized by sentinel bacteria after the improvement phase.

Tay (2016) and Tay et al. (2017) conducted detailed studies of lean projects in two large hospitals. They found the redundancy between resource efficiencies and flow efficiencies, and identified key contextual factors that drove the project orientation to each of them. Tay et al. (2017) defined the factors as: service variety, interdependence, capital resource intensity and service exclusivity. They proposed a conceptual framework and four propositions that integrate the contextual factors to determine the dominant focus on lean projects. Afterwards recommendations are made about how the efficiency paradox can be avoided.

Amran et al. (2020) verified factors that cause barriers in the implementation of lean healthcare in one government service sector in Malaysia. Tortorella, Dun, \& Almeida (2020) conducted an extensive literature review with a longitudinal comparison study to identify leadership behavior in the middle manager of a health institution that adopted lean healthcare.

\subsection{Surgical tray optimization}

Surgical tray optimization reduces hospital costs and improves the efficiency of the surgery team. Some authors carry out research in the area, such as those described below.

Furthermore, with the same lean purpose of eliminating/reducing activities that do not add value to the patient, professionals or the hospital, the European Study Group with Industry (ESGI) evaluated a Portuguese public hospital in order to analyze the pattern of use of surgical instruments from the grouping of instruments that comprise each case, taking into account the maximum advantage of the available equipment and the minimization of material sterilization cycles. Besides, it was the purpose of the study to identify groups of materials to be included in packaging or surgical boxes based on the evaluation of the minimum difference in the use profile (high internal consistency) and the significant differences between different groups of boxes (high external heterogeneity, or that is, between groups of boxes. To achieve these objectives, Alves et al. (2016) proposed combinatorial, statistical and grouping tools to optimize the composition of surgical kits.

Byrnes, Schmitt, Tommaso, \& Occhino (2017) evaluated the use of vaginal hysterectomy trays to reduce unnecessary instruments without compromising high-quality and safe patient care. They managed to reduce it to 48 instruments in the minor gynecology tray, considering the initial scenario of 
the standard vaginal hysterectomy tray with 67 instruments. The cost savings in the department have been modest, but the biggest advantages include reduced instrument setup and processing times and instrument longevity.

Cichos et al. (2019) assessed the economic impact of optimizing orthopedic instrument trays at a tertiary medical center. They applied the lean methodology to analyze twenty-three trays of independent orthopedic surgical instruments in a single academic hospital. After the analysis, the surgeons agreed on the lowest number of instruments for the procedure trays.

Crosby et al. (2019) quantified the potential time savings associated with surgical tray optimization. The use of an instrument for the procedures was reviewed: septorhinoplasty, sinus surgery, tonsillectomy, and septoplasty, from the Department of Otolaryngology at St Joseph's Health Care, London. It has been found that tray optimization can reduce adverse events, stress and improve management of estimated personnel needed. However, it was not possible to determine the costbenefit due to little understanding of how the redistribution of time savings is given by departments.

Farrelly et al. (2017) conducted a single-site observational study in a large academic medical center. They found that surgical tray optimization allowed benefits such as reduced material costs, reduced labor required to count, pack and decontaminate surgical trays, relatively simple improvement of the microsystem, which can generate a significant reduction in hospital costs. It can also reduce costs associated with processing surgical instruments in a large multi-hospital academic center.

Marchand et al. (2020) compared traditional surgical trays used for total knee arthroplasties (TKAs) procedures and sealed with sterile containers in terms of: processing time of the trays in the central sterile supply (CS); operating room (OR) and CS costs and waste produced; preparation and cleaning time in the OR. The use of optimized trays and sealed sterilization containers reduced the number of trays by three and the turnover time, on average, by 57 minutes. The annual savings in processing CS and OR was estimated at $\$ 249,245$. Waste disposal has been estimated at 10,590 ounces for traditional containers and 450 ounces for sealed containers.

\subsection{PDCA}

According to Worth et al. (2012), the PDCA cycle is applicable both in the clinical scenario itself and in the improved scenario, since the changes are driven by the flow of value. Wang (2014) applied the PDCA in medical record archiving management, Liu et al. (2014) used it to manage the quality of these records in the orthopedic department and Chen and Lu (2014) analyzed the effects of using the PDCA cycle applied to the quality management of medical records, obtaining satisfactory results, since the hypothesis is that the quality of the medical record can be significantly improved by handling the PDCA and this is statistically proven. 
In this sense, this work was conducted according to the following steps:

(1) Plan: the behavior of standard spinal treatments in the view of neurosurgeons and orthopedists was analyzed, and the current situation was evaluated in detail considering the need to change by means of the VFM of the current and future situation.

(2) Do: a plan was devised to implement changes through a series of experiments with the application of the $5 \mathrm{~S}$ tool in the elaboration of the surgical kits.

(3) Check: it was proposed to implement the changes, using Kaizen and the tools of standardized work and visual management.

(4) Act: it is suggested that appropriate follow-up actions are undertaken.

\section{Materials and methods}

The proposition of this research is based on the fact that Lean Healthcare tools can help in the scale of instrumental kits needed for two types of spine treatments: Percutaneous Facet Rhizotomy and Medullary Stimulation Therapy or Medullary Neurostimulation. This proposition is motivated by the authors' arguments presented in section 2.

To achieve the proposed goal, the research approach used was qualitative and the procedure was a case study. For Creswell (2014) the qualitative approach consists of techniques for describing, decoding, translating and learning the meaning of naturally occurring phenomena. According to Bryman (1989), it is considered a mistake to interpret that the difference between the quantitative and qualitative approaches is the lack of quantification in the later. Qualitative research tends to be less structured, in order to capture the perspectives and interpretations of the object of study, which cannot be interpreted as less rigorous. The qualitative approach has no established forms for data analysis, unlike the quantitative approach, which uses structured forms such as statistical methods to analyze collected data (Martins, 2012).

The case study as a research procedure allows researchers to obtain knowledge of general and significant characteristics of real events (Yin, 2015). The use of this approach and procedure is justified by the description and learning of a real process. In this case, the process of spine surgery was the focus, obtaining knowledge and proposing changes and contributions to it.

In conducting the study, a problem-solving method based on the PDCA cycle was used. The necessary data involving all the aforementioned steps were obtained through semi-structured and unstructured interviews with the employees of a private hospital for approximately four months. The personnel involved in data collection encompasses professionals in the areas of surgical instrumentation, hospitalization, treasury, audit, SC and medical surgeons specializing in neurosurgery 
and orthopedics. Thus, the entire dimensioning carried out was defined jointly with the professionals directly involved with the operations and uses of the kits.

The method used in the analysis of qualitative data collected was content analysis. According to Bardin (2006), this method encompasses a set of information analysis techniques in order to critically understand the explicit and hidden meaning and significances of the analyzed data. In order to do this, the data analysis process is hierarchized in three stages, pre-analysis, material exploration and data treatment, inference and interpretation, therefore condensing and highlighting the information for analysis, culminating in inferential interpretations (moment of intuition, analysis reflective and critical).

In the Plan step, an employee from each of these sectors and a physician from each of the abovementioned fields were interviewed, so that the current process and the VFM could be designed, for which the procedures' phases were discussed, starting from the patients' medical consultation until the post-surgical hospital discharge, the average time spent in each of these phases, the personnel, the companies and the materials involved. In the Do step, specific data were collected for assembling the kits, such as the necessary instruments, the sterilization procedure and time or disposal and storage. As for the Check and Act steps, we made some suggestions regarding the deployment and follow-up, since these exceed the scope of this research.

\section{Results and discussion}

Among the spine treatments area, Percutaneous Facet Rhizotomy and Spinal Stimulation Therapy or Spinal Neurostimulation were chosen as the focus of this work. The two procedures are used as a treatment for back pain; however, they work differently.

The spinal cord stimulator allows the control of chronic pain, since conventional procedures are not successful. For example, a patient who has chronic low back pain and has already undergone procedures that involve clinical treatments with the use of medications and even surgical ones, such as lumbar rhizotomy, may be subjected to the implantation of the generator, which produces an electrical current that is transmitted to specific spinal cord poles and therefore, the pain ceases. Facet rhizotomy is also applied to chronic pain. It is a neurolysis that is, a thermal lesion of the affected facet, decreasing its sensitivity and, consequently, pain.

\subsection{Plan}

At this stage the procedures that should be studied and their design for a better understanding are defined. The criterion of choice was to analyze a high complexity procedure instead of simple procedures or general surgeries. Therefore, we chose spinal treatments that involve a high degree of knowledge and the opinion of specialists in different fields. 
After defining the procedures to be studied, the Value Stream Mapping of the current situation was performed to identify the shortcomings of the process and the VFM of the future situation, in order to propose, along with other lean tools, an improvement plan.

\subsubsection{Definition of procedures and VFM of current and future situations}

Among the area of spinal treatments, Percutaneous Facet Rhizotomy and Medullary Stimulation Therapy or Medullary Neurostimulation were chosen as the focus of this study. Both procedures are used as a treatment for back pain, but they act in different ways.

The medullary stimulator allows the control of chronic pain, as conventional procedures are not successful. For example, a patient who has chronic low back pain and has undergone procedures that involve clinical treatments with the use of medications and even surgical ones, such as lumbar rhizotomy, can be submitted to generator implantation, which produces an electric current that is transmitted to specific poles of the spinal cord, therefore ceasing pain. Facet rhizotomy, also applied to chronic pain, is a neurolysis, that is, a thermal lesion applied to the affected facet, reducing the sensitivity of the facet and, consequently, the pain.

The chosen processes are elective procedures, that is, they must be pre-scheduled for their execution and include the following phases:

(1) The patient with low back pain, after performing other unsuccessful treatments, consults with the orthopedist or neurosurgeon in order to find alternatives for the solution of his/her problem. The physician then recommends Percutaneous Facet Rhizotomy or Spinal Cord Stimulation therapy, depending on the case, and makes the request for the procedure;

(2) Considering that the patient has health insurance, the request must be sent to the insurance company for authorization;

(3) After the request authorization is approved by the insurance company, the Department of Orthotics, Prostheses and Special Materials (OPSM) lists the materials to be used and their prices to the supplier;

(4) Parallel to phase 3, the patient should contact the physician's secretary to set the date of the procedure. This date is defined according to the availability of the physician and the SC, in accordance with the schedule of the hospitalization sector;

(5) In the timeframe between the authorization and the date of the procedure, the patient performs preoperative examinations and is submitted to a consultation with the anesthesiologist to receive instructions and guidelines;

(6) Once the quotation (i.e. price assessment) is made, the necessary material is requested, which has up to 24 hours before the surgery to be delivered by the supplier; 
(7) When the material arrives at the hospital, it is checked and sent to the SMC;

(8) At the SMC the materials' sterilization process is carried out, when applicable;

(9) The instrumentator assembles the kit with the related materials, so the procedure can be performed;

(10) Hours prior to the procedure, once the type of anesthesia is determined, which is usually general anesthesia for the Medullary Stimulation Therapy and light sedation with local anesthesia for Percutaneous Facet Rhizotomy, the pharmacy assembles the medication kit for the anesthesia and anything else needed, and send it to the SC;

(11) The neurosurgeon or orthopedic physician performs the procedure;

(12) After the procedure, the patient is referred to a recovery room to recover from anesthesia;

(13) After the patient recovers from the anesthesia, he is taken to the room where he will be admitted for recovery;

(14) After the hospitalization period, the patient is discharged and the recovery process continues at home, with periodic returns, among other procedures recommended by the attending physician.

Figure 1 simplifies the 14 phases of the elective procedure presented.

Figure 1

Stages of the Elective Procedures

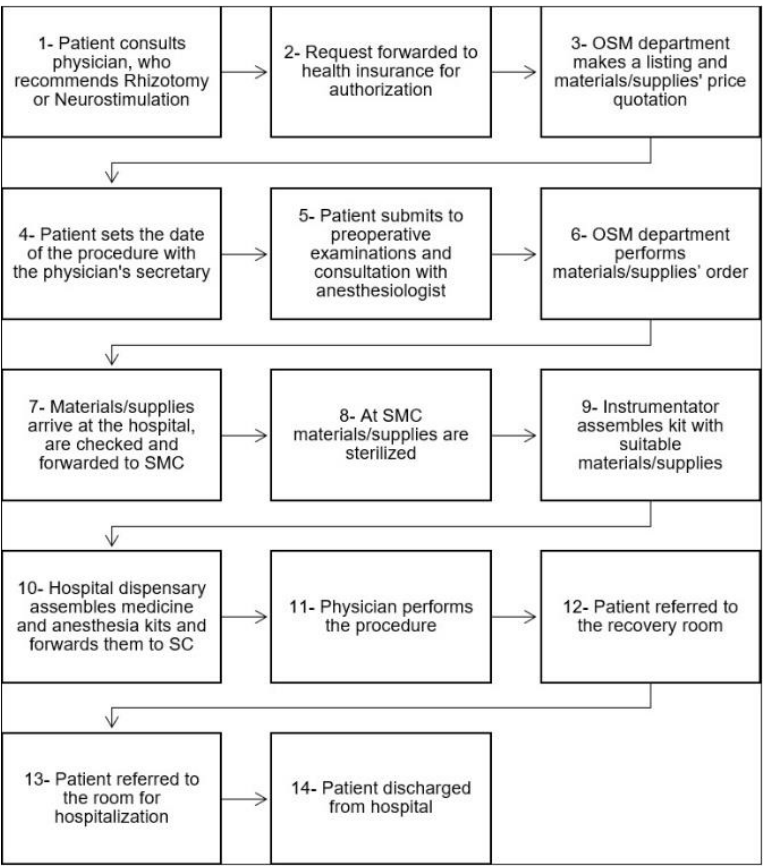

Source: Research data.

Based on the described process and the other data collected, it was possible to design the VFM of the spinal treatment processes' current situation, shown in Figure 2. Analyzing the current situation 
map, we notice that the elapsed time between the materials' listing and its availability in the SC occurs in up to six days, this time varying according to the material used by each doctor, to the availability of the OPSM department to carry out the prices' quotation, to the delivery time of the supplier and to the need and frequency of material sterilization, and when deemed necessary, the resterilization of those that were already stocked in the hospital warehouse.

Still, the patient, who is the client of the process, does not know or do not have direct contact with all the activities described, which reduces the comprehension of the importance of these for the whole procedure. The application of lean tools allows the elimination of activities that do not add value to the client of the main flow and reduces the time and frequency of those that do not add value, but are necessary.

It can be seen in Figure 2 that the processes are executed sequentially from the medical appointment and that the total lead time is 21 days. Also, the PDCA Cycle, 5S, Kaizen, Standardized Work and Visual Management were applied when analyzing the processes between and including materials/suppliers 'listing and Medication's kit assembly, where it was possible to propose management improvement actions.

In the VFM of the future situation shown in Figure 3, the activities that did not add value in the client's vision were eliminated from the main flow. This reduced the patient's waiting time by one week. In order to create a parallel flow with these activities, the use of lean production tools such as 5S, standardized work and Kaizen, are discussed in sections 4.2 and 4.3.

Note that the total lead time was reduced to 16 days ( 21 days in the current situation - Figure 2), representing a possible percentage reduction of $23.81 \%$, if the hospital applies the proposed improvement suggestions. This reduction was possible due to the reorganization of the processes, where the opportunity to carry out activities in parallel was identified, considering they originally were all performed sequentially from the patient's medical appointment. It was also possible to eliminate the stock between the medication's kit assembly and the surgical procedure. 
At this stage happens the planning of the surgical kits' assembly. As the $5 \mathrm{~S}$ define the visual control in an organization and allows it to develop a classification, order and cleaning planning to increase productivity, safety, employee motivation, organizational Atmosphere and competitiveness improvement in order to eliminate waste, so it will be used as a support tool in this step.

\section{Figure 2}

VFM of the Current Situation of the Spinal Treatment Processes

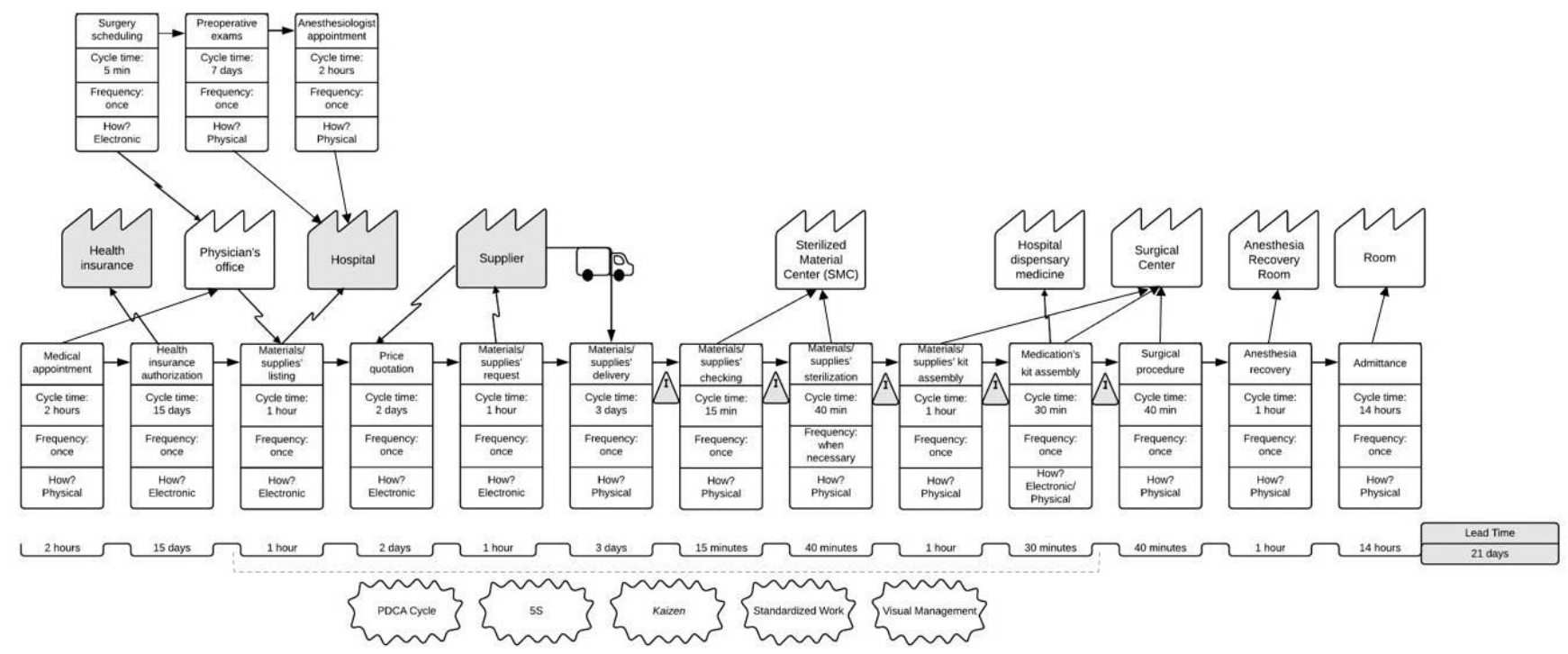

Source: Research data. 
Figure 3

VFM of the Future Situation of the Spinal Treatment Processes

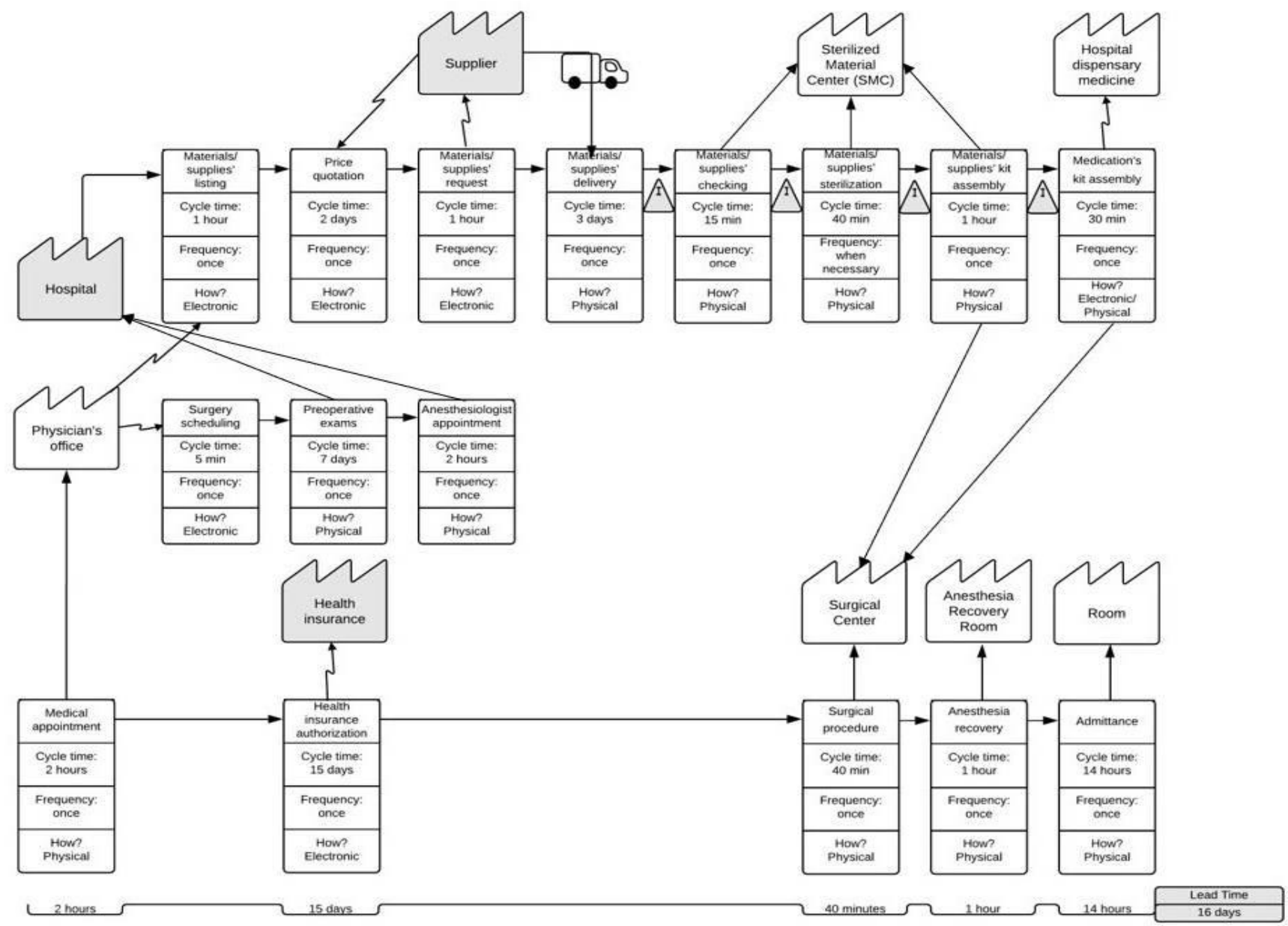

Source: Research data.

\subsubsection{Application of $5 \mathrm{~S}$ tool in the elaboration of surgical kits}

The standard of spinal treatments considering the points of view of neurosurgeons and orthopedists alters only due to the thoroughness of the procedure. Neurosurgeons are more meticulous because they operate more sensitive regions of the human body, like the brain. This requires the use of microscopes in most surgical procedures and hand tremors and any kind of failure are inadmissible. On the other hand, orthopedic surgeons deal with coarser parts such as bones, constantly using heavy tools like hammers and drills.

Nevertheless, in the case of Percutaneous Facet Rhizotomy and Medullary Neurostimulation Therapy, the materials used by both specialties are the same. Therefore, the configuration of the kit for these types of treatments becomes simpler. The $5 \mathrm{~S}$ tool will be applied in this context. 
In the first step of the 5S ("Seiri" - separate) tool the necessary items and the packaging used for storage in the SMC were separated for each procedure. This is part of the OPSM department's responsibility.

The necessary tracking of the items for Percutaneous Facet Rhizotomy are radiofrequency generator, cannula kit for rhizotomy, neurotomy (electrode), and stainless-steel tray. The items used in Medullary Neurostimulation Therapy are scopia, basic lumbar box, bipolar scalpel, generator for spinal cord neurostimulation, puncture catheter kit, neuro-sensitive stimulation catheter kit, non-woven SMS fabric, and stainless-steel tray.

The listed kits (rhizotomy cannula kit, puncture catheter kit and neuro-sensitive stimulation catheter kit) are already assembled from the manufacturer, containing the needles and catheters needed for the indicated procedures.

In the second "S" ("Seiton" - simplify or adjust) the ordering and classification of the listed material took place, considering what is equipment, what is sterilizable or disposable material, what is packaging, what is the expiration date, how much of it is valid, where it should be stored and the organization of the sterilizable material. Tables 2 and 3 illustrate some of these data in an organized way. In Table 2 there are items used in Percutaneous Facet Rhizotomy, their classification and storage location. Table 3 brings the items classifications referring to the material used in Medullary Neurostimulation Therapy.

\section{Table 2}

Classification of the Necessary Items for Percutaneous Facet Rhizotomy

\begin{tabular}{l|l|l}
\hline \multicolumn{1}{c|}{ Item } & \multicolumn{1}{c}{ Classification } & \multicolumn{1}{c}{ Storage } \\
\hline Radiofrequency generator & Equipment & Restricted sterile site \\
\hline Cannula kit for rhizotomy & Disposable material & Restricted sterile site \\
\hline Neurotomy (electrode) & Disposable material & Restricted sterile site \\
\hline Stainless steel tray & Sterilizable packaging & Restricted sterile site \\
\hline
\end{tabular}

Source: Research data.

The electrode may be disposable or sterilizable according to the manufacturer. In the first case, it has the same expiration date as the needles in the cannula kit, meaning they are valid for up to three years depending on the manufacturer if the original factory packaging is intact. In this study, only disposable electrodes were considered for the assembly of the rhizotomy kit. 
Table 3

Classification of Items Needed for Medullary Neurostimulation Therapy

\begin{tabular}{l|l|l}
\hline \multicolumn{1}{c|}{ Item } & \multicolumn{1}{c}{ Classification } & \multicolumn{1}{c}{ Storage } \\
\hline Scopia & Equipament & Restricted sterile site \\
\hline Basic lumbar box & Sterilizable material & Restricted sterile site \\
\hline Bipolar scalpel & Sterilizable material & Restricted sterile site \\
\hline Generator for spinal cord neurostimulation & Sterilizable equipment & Restricted sterile site \\
\hline Puncture catheter kit & Sterilizable material & Restricted sterile site \\
\hline Neuro-sensitive stimulation catheter kit & Sterilizable material & Restricted sterile site \\
\hline Non-woven SMS fabric & Disposable packaging & Restricted sterile site \\
\hline Stainless steel tray & Disposable packaging & Restricted sterile site \\
\hline
\end{tabular}

Source: Research data.

The non-woven SMS fabric used has a sterilization expiration date of up 90 days. This means that from the moment the material is sterilized and stored with the aid of this "fabric", its sterilization keeps valid for three months, as long as it is kept in a restricted sterile place.

In the sense of cleanliness and zeal ("Seiso" - cleaning), in order to identify and ensure that the items and equipment are ready for work, there is the sterilization of the sterilizable material, the organization of the items in the stainless tray and the wrapping of the tray with the SMS fabric. When it comes to sterilized material, the external identification of the kit is done with a permanent pen. Data regarding material sterilization are given in Table 4.

Table 4

Material Sterilization Data

\begin{tabular}{|c|c|c|}
\hline Sterilizable material & Sterilization Time & Temperature \\
\hline Basic lumbar box & 33 minutes & $134 \div \mathrm{C}$ \\
\hline Bipolar scalpel & 40 minutes & $121 ㅇ ㅡ$ \\
\hline Generator for spinal cord neurostimulation & 40 minutes & $121^{\circ} \mathrm{C}$ \\
\hline Puncture catheter kit & 40 minutes & $121 \stackrel{\circ}{ } \mathrm{C}$ \\
\hline Neuro-sensitive stimulation catheter kit & 40 minutes & $121 ㅇ ㅡ$ \\
\hline
\end{tabular}

Source: Research data.

To maintain the previous three "S" features ("Seiketsu" - standardize), standardization is required. To this end, it is suggested to include the surgical kits for Percutaneous Facet Rhizotomy and Medullary Neurostimulation Therapy in the Medication and Materials Standardization Book, according to the items in Tables 4 and 5 . Therefore, the sterilizable material is sterilized and the disposable material verified to see if their packaging is intact, with needle sizes already defined and separated according to the region of the spine where the surgery will take place (lumbar, cervical or thoracic), as well as containing the material for packaging and identification. 
According to the demand of each type of treatment, it is necessary to stock a certain quantity of kits. Therefore, to standardize this process, one must quote the price of the material and buy it, so that a safety stock is maintained in order not to lack materials for treatments of these nature.

All staff involved in the process should be aware of the deployment of the new kits. From OPSM staff, SMC, SC, nurses, technicians and physicians so that nobody requests materials that are not available in the kits or specified in the standardization book for these types of procedures.

The materials used in the surgical kits for the Percutaneous Facet Rhizotomy and Medullary Neurostimulation Therapy procedures are classified as Special Materials. These are materials and devices for individual use that assist in diagnostic or therapeutic procedures and that do not fit the specifications of orthoses or prostheses, which may or may not undergo reprocessing, according to rules determined by the Brazilian National Health Surveillance Agency (Anvisa). They are the responsibility of the OPSM department. This department is responsible for controlling these materials in addition to the SMC, which is a technical support sector, whose purpose is to provide these materials properly processed, providing conditions for service (Ministério da Saúde, 2016).

The fifth "S" ("Shitsuke" - support) proposal merges with the ideas of the Check and Act steps of the PDCA Cycle in this research. This regards the development of habit and discipline to maintain the first four " $\mathrm{S}$ ". The subsequent stages of the cycle use the Kaizen, the standardized work tools and visual management to propose the implementation and monitoring of the proposed changes.

\subsection{Check}

At this stage, it was proposed the application of the Kaizen tools, standardized work and visual management for the development of the support feature.

Respecting the three pillars of standardized work (takt time, work sequence and standard stock in process), it is proposed to standardize and maintain the spinal treatments process with the use of selected instrumental kits. In this standardized work, the expected logical sequence follows the VFM of the future situation, as presented in Figure 3. The standard stock obeys the items described in the Standardization Book of Medications and Materials and each involved team member must be willing to follow the guidelines and be aware of their responsibilities. This ensures patient reliability and safety within the takt time admissible and comfortable to him.

In order to maintain the proposed changes, it is suggested to implement the Kaizen associated to the visual management tool, at first, with the focus on the employees' awareness of the advantages brought with the implementation of the new system. These ensure improvement with the involvement of all, which is the basic Kaizen concept. In order to present the proposal of the kits, the medical, technical and administrative staff involved in the processes of the studied treatments, it is advisable the 
execution of a (kind of) workshop involving visual identities, which catches the attention and make the employee, when walking down the corridor of the hospital, to stop and take notes about the new system. The employee's invitation can be done via email, for example.

In the workshop, it is recommended to display assembled, packaged and identified kits, the hospital's Standardization Book of Medications and Materials along with a manual on how to use or access it and a SC manager to whom direct questions and provide contributions to interested parties. For the purpose of visual communication, it is advisable to use posters, banners, popup trade shows (i.e. tents bringing images and informative texts), as well as handbooks or simple brochures for those who wish to take additional information. In addition to this, a strategic location is important considering this busy environment where time is precious. The establishment of points placed near more calm or informal places, such as the corridor near the doctors' rest room, the pantry, kitchen or mess hall where the snacks are made. Also important is to open a space for expressing suggestions, opinions and improvements.

4.4 Act

The suggestion for follow-up actions focuses on maintenance, to support the application of standardized work and Kaizen, making them part of the company's culture, it is suggested, along with the development of new internal policies for the use of the surgical kits, a Human Resource Management (HRM) (Hendry, 2012) and programs to reduce resistance to change and to determine the minimum stock of surgical kits. Given that prominent companies have their culture focused on performance and innovation, which, in addition to being a critical success factor, is increasingly becoming a survival criterion for organizations and having it requires the audacity to develop a process or new product to the detriment of an old one, demanding commitment from all those involved.

For the application of surgical kits there will be a break from old organizational patterns, the socalled breakthrough, which has led organizations to focus their efforts on intellectual capital, providing them with competitive intelligence. In this sense, HRM should work to facilitate the implementation of new surgical kits Internal Policies, by means of training and continuous learning, aiming at a solid organizational culture with alignment of values and principles.

However, regarding organizational culture, hospital institutions are considered quite conservative. Health professionals tend to accept innovations for care, such as new equipment and technologies for diagnosis and therapy. Besides, these professionals are considered highly specialized, having a greater identity with the technique than with the institution, which leads to different lines of authority: a technical and an administrative one, in which managers have little effective control over the physicians. 
In order to overcome the barriers that hinder the implementation of new systems and even the development of a new organizational culture, it is proposed to use the 3Cs (Grosz, 1996), which are about collaboration, communication and coordination. To the extent that new policies, systems, processes, objectives, norms, and all variables that influence business behavior emerge, efficient communication methods must be developed to disperse information, ask questions, propose improvements, considering that at least one of the communication channels used should be bilateral, so that base employees can have contact with the planning and management core, to expose the real needs of the patients and servants who deal daily with the difficulties in attendance.

Communication is the basis of the 3Cs. By applying it, cultural coordination is developed and maintained, since the more efficient the communication systems, the greater the learning of the internal culture and the organizational control and coordination capacity. As a communication channel, it is suggested the use of a wall newspaper or a corporate television to provide agile communication to employees about the company's business, HR, health, social responsibility, among others, and an intranet as a channel for employee relations of all levels, with the transmission of corporate and organizational information.

As for programs, aimed to reduce resistance to change, communication is highlighted, but in this case, individual discussions to study the employee's points of view and education is indicated with the use of memoranda, reports and presentations in small groups. Employee attendance and involvement in decision-making is essential for reducing resistance, i.e., informing, listening, asking questions, proposing and accepting employee improvements throughout the transformation process, not only after deployment. In the most extreme cases of resistance, methods such as facilitation aimed at the breakdown of fears and anxieties by means of counseling and training are suggested. In addition to this, negotiation, when the source of resistance is powerful, manipulation, to gain support and credibility or even coercion, which uses direct threats to the resistant are options to be considered.

For the determination of the surgical kit for Percutaneous Facet Rhizotomy and for Medullary Neurostimulation Therapy minimum stock, it is suggested that a more rigorous procedures' control of this type be performed in the hospital SC, considering all the necessary information and documenting them in the hospital's computer system. As doctors working in private clinics and other hospitals also operate in the hospital's SC, the procedures performed by them should be better accounted for, since the material is requested from the hospital's OPSM department.

In order to have this internal control, it is recommended that each procedure is coded as a product, for example Percutaneous Rhizotomy of Lumbar Facets will obtain the code "01-PRLF " and every database related to it will be fed this code, whether in the warehouse, the hospitalization, the OPSM department or in any sector of the hospital. Thus, the control of the procedures demand in the $\mathrm{SC}$ will be more efficient and effective, as well as the control of the material in the inventory. 
From this observed demand, it will be possible to define the minimum stock of each type of surgical kit. As for health services, demand will probably be characterized as irregular, without explicit seasonal or trend patterns. In this case, procedures with irregular patterns should be separated from those that have regularity, using simple forecasting methods such as exponential weighting or an annual regression technique (Ballou, 2007). From this demand forecast, the number of necessary kits of each type in stock can be obtained, guiding the purchase function and avoiding the lack of material at the time of the procedure.

In order to calculate the prediction using exponential weighting, for example, a simple expression is used, which considers only the most recent period forecast, the actual demand of the current period and the exponential weighting constant $\alpha$. In the case of irregular demand, the use of a low value for $\alpha$ (between 0.01 and 0.3 ) is indicated, which gives greater weight to the historical demand in the future forecast, making the forecast more stable, not reacting quickly to changes in demand levels. Equation 1 presents the expression to calculate the demand forecast for the next period (Ballou, 2007).

New forecast $=\alpha$ (current demand $)+(1-\alpha)$ (previous demand $)(1)$

For example, consider the need for ten kits for Lumbar Facet Percutaneous Rhizotomy for the current month. However, the real demand was six units. The value considered for the weighting constant was 0.3. The expected value for next month's demand, according to Equation 2, would be:

New forecast $=0.3(6)+(1-0.3)(10)=8.8(2)$

That is, approximately nine kits must be in stock for the next month. This demand will be used as "previous demand" for the calculation in the next period and the OPSM department will purchase the necessary material for the assembly of six Percutaneous Lumbar Facet Rhizotomy kits for the next month. Since the previous forecast indicated the purchase of ten and six were used, four remained in stock and five are missing to complete the amount of the new forecast.

Thus, in summary, the propositions for the company were:

(1) Development of an HRM with the use of its techniques both internal and external to the hospital environment;

(2) Use of the five basic processes for people management in HRM maintenance;

(3) Implementation of new Internal Policies with the use of the 3Cs (culture, communication and coordination);

(4) Coding of procedures such as products and database feeding by means of codes;

(5) Definition of minimum surgical kit stock of each type using exponential weighting. 


\subsection{Summary of the methodological procedure}

The summary of the entire methodological procedure performed to achieve the results of this article is presented in Figure 4.

\section{Figure 4}

\section{Summary of the Methodological Procedure}

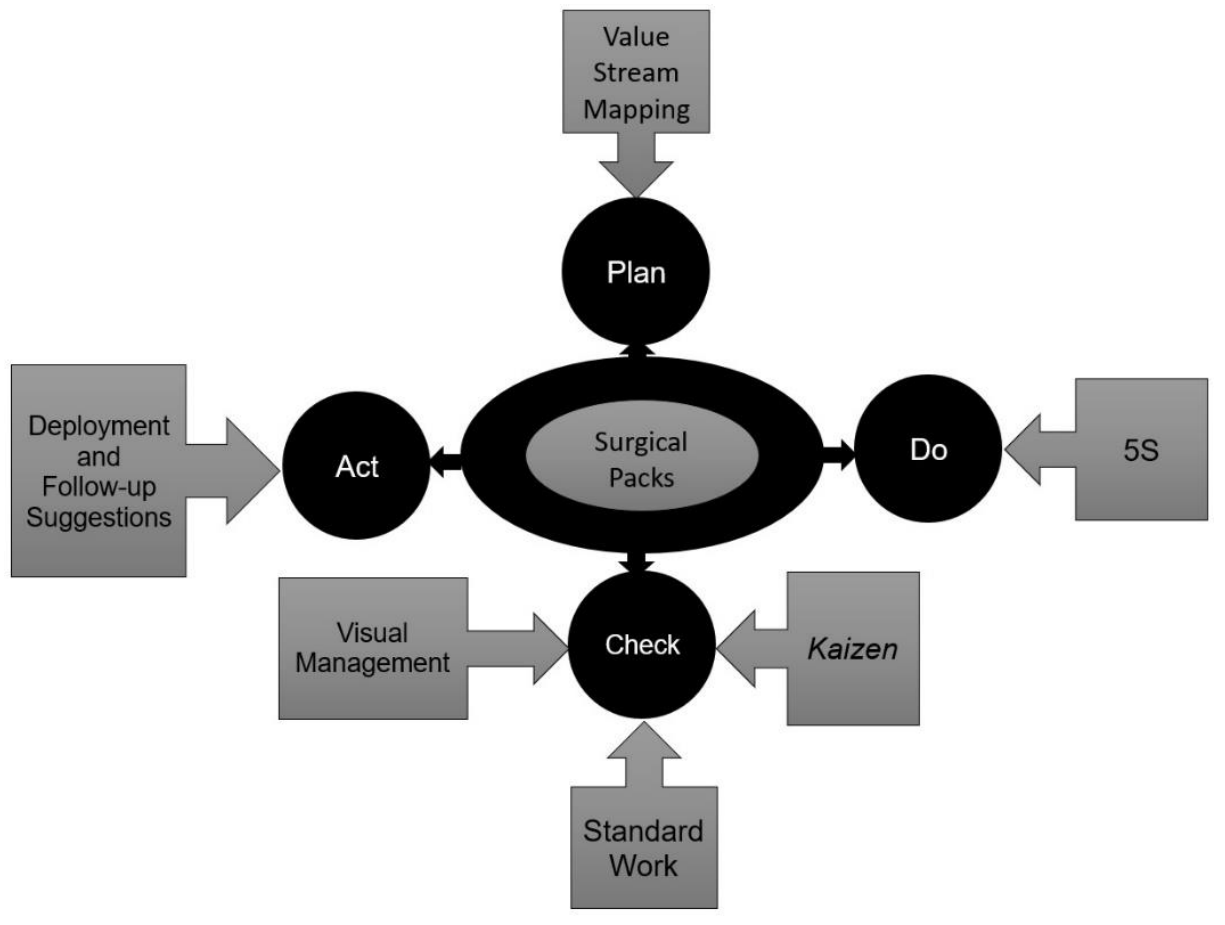

Source: Elaborated by the authors.

As in this work, the value flow mapping was used by Wannemuehler et al. (2015) in the "Plan" stage to determine the use of surgical instrumentation and the reprocessing workflow for performing adenotonsillectomies. Research on the use of pre-intervention instruments allowed a consensual and intelligent reduction of the set of instruments. According to the authors, the reduction in the instruments set is ideal for eliminating waste due to the accumulation of tools over time and the obsolescence of the instrument as new technologies are adopted.

In the "Do" phase, $5 S$ was used as in the work of Farrokhi et al. (2015). This lean tool was selected to improve the quality and efficiency of instrument availability in the operating room, from classification (determining the use and waste of the instrument), simplification (removing unnecessary instruments), scanning (confirming the availability of the necessary instruments), standardization (all the same trays for a given procedure) and self-discipline (monitoring success). The primary outcome 
was the reduction of unnecessary instruments delivered to the operating room. As a secondary analysis, a reduction in surgery times was achieved.

In his book "Kaizen workshops for lean healthcare", Jackson (2012) states that kaizen is the basis of all lean healthcare methodologies. According to the author, to maintain continuous improvement, kaizen workshops should be held periodically as proposed in this work in the "Check" stage and can also be used in the "Act" stage as a follow-up action.

Still in the "Check" stage, regarding visual management, Mannon (2014) presents the experience of ThedaCare, which started its transformation in five hospitals and 27 medical clinics with the use of lean healthcare in 2003. To communicate the transformation and, also, maintain it, insight management was used during and after the whole process. Regarding standardized work, ThedaCare used this lean principle in all steps of patient care processes in treating pneumonia, serious infections, heart problems, and other issues. According to Mannon (2014), quality components were integrated into standardized work.

As for the proposal for the use of Equation 1 to calculate the demand for assembling the kits, proposed by (Ballou, 2007), it is noted with the study of the literature, that authors have used more robust techniques to perform this function, as in Alves et al. (2016).

\section{Conclusion}

The health field manifests particularities that make it difficult to manage. In this article, concepts and techniques from manufacturing, such as lean production, called Lean Healthcare in this context, were used to assist in hospital administration. This approach has been addressed by several authors and, in this work, is focused on the proposal to implement surgical kits for spinal treatments in order to reduce time, costs, errors and provide safety to the medical staff and, especially, the patient.

Instrument kits were devised for two types of spinal treatments using Lean Healthcare tools as support. We proposed kits containing surgical material to perform Percutaneous Facet Rhizotomy and Medullary Stimulation Therapy, using tools such as VFM, Kaizen, visual management, 5S, standardized work and the PDCA cycle as a problem-solving method.

For this, the behavior of the standard spinal treatments in the view of neurosurgeons and orthopedists was analyzed, concluding that the material used by both is the same, differentiating only in terms of procedure minutiae. Tools of standardization, continuous improvement and visual management were applied in the verification step to propose the implementation and monitoring of the use of the kits. It was proposed to implement the surgical kits based on internal standardization policies and actions were suggested to follow the proposed improvements such as the development of 
an HRM, programs to reduce resistance to change and methods for determining the minimum stock of surgical kits.

In case the studied hospital duly implements the results of this research, it will be possible, among other benefits, to eliminate the waiting time of the patient to perform the procedure after he is approved by his health insurance. This reduces the current waiting time by one week, with the elimination of activities that do not add value to the customer's vision. For the medical staff, the entire hospital, the patient's family, and the patient him/herself, surgical instrument kits are a guarantee of safety in performing the procedure, since all the necessary material will be available at the right time, in the correct quantity, assuring agility, speed and hygiene.

The article depicts the Lean Healthcare approach, which can be used from the decision support standpoint to the implementation and monitoring of practical improvements. This contributes in the academic scope, by further disseminating the use of the research topic in practical and business situations, since the hospitals' managers can use this research as an aid in the decision-making processes in terms of sizing the surgical kits. Among the practical contributions of the possible implantation of kits by the hospital are: reduction of the number of instruments for the procedure trays, reduction of adverse events, reduction of stress, better management of estimate of necessary personnel, reduction of the cost of materials, reduction of the work required to count, pack and decontaminate surgical trays. Kit standardization is a simple improvement of the microsystem, but can decrease hospital costs, such as costs associated with processing surgical instruments.

For society, this research contributes to reducing the lead time of the complete process, allowing patients to receive the necessary surgical treatments more quickly, improving the well-being of these patients and the better visibility of the activities of the total process by the patient. Still, the suggested methodological procedure can be adapted and used by other hospitals.

As future research, we suggest the follow-up on the deployment of the proposed kits in the target hospital routine and the design of other kits for other surgical procedures and medical specialties.

\section{References}

Adebanjo, D., Laosirihongthong, T., \& Samaranayake, P. (2016). Prioritizing lean supply chain management initiatives in healthcare service operations: a fuzzy AHP approach. Production Planning \& Control, 27 (12), 953-966. doi: https://doi.org/10.1080/09537287.2016.1164909.

Alves, A. C., Gonçalves, A. M., Fernandes, J. M., Vaz, I., Teixeira, S., Sousa, I., Pereira, J. J., \& DóriaNóbrega, S. (2016). Combined tools for Surgical Case Packages contents and cost optimization: a preliminary study. Procedia Computer Science, 100, 393-398. doi: https://doi.org/10.1016/j.procs.2016.09.174

Amran, M. D. M., Januddi, F., Nuraina, S., Ikbar, A. W. M, \& Khairanum, S. (2020). The barriers in lean healthcare implementation. Test Engineering and Management, 82 (1-2), 1972-1981. 
Ballou, R. H. (2007). Business logistics/supply chain management. India: Pearson Education.

Boronat, F., Budia, A., Broseta, E., Ruiz-Cerdá, J. L., \& Vivas-Consuelo, D. (2018). Application of Lean Healthcare methodology in a urology department of a tertiary hospital as a tool for improving efficiency. Actas Urológicas Españolas (English Edition), 42 (1), 42-48. doi: https://doi.org/10.1016/j.acuroe.2017.11.008.

Bryman, A. (1989). Research methods and organization studies. London: Uniwin Hyman.

Byrnes, J. N., Schmitt, J., Tommaso, C., \& Occhino, J. A. (2017). Cost reduction techniques in the operating suite: Surgical tray optimization. American Journal of Obstetrics \& Gynecology, 216(3), Supplement, S616, March 01. doi: https://doi.org/10.1016/j.ajog.2016.12.119.

Chen, M., \& Lu, Z. (2014). Effects of PDCA Applied to Medical Record Quality Management. Chinese Medical Record English Edition, 2(8), 372-375. doi: https://doi.org/10.3109/23256176.2014.992175

Cichos, K. H., Hyde, Z. B., Mabry, S. E., Ghanem, E. S., Brabston, E. W., Hayes, L. W., McGwin Jr. G., \& Ponce, B. A. (2019). Optimization of Orthopedic Surgical Instrument Trays: Lean Principles to Reduce Fixed Operating Room Expenses. The Journal of Arthroplasty, 34, 238-2840. doi: https://doi.org/10.1016/j.arth.2019.07.040.

Collar, R. M., Shuman, A. G., Feiner, S., Mcgonegal, A. K., Heidel, N., Duck, M., Mclean, S. A., Billi, J. E., Healy, D. W., \& Bradford, C. R. (2012). Lean management in academic surgery. Journal of the American College of Surgeons, 214(6), 928-936. doi: https://doi.org/10.1016/j.jamcollsurg.2012.03.002.

Costa, L. B., \& Godinho, M., Filho. (2016). Lean healthcare: review, classification and analysis of literature. Production Planning \& Control 27 (10): 823-836. doi: https://doi.org/10.1080/09537287.2016.1143131.

Costa, L. B., Godinho, M., Filho, Rentes, A. F., Bertani, T. M., \& Mardegan, R. (2015). Lean healthcare in developing countries: evidence from Brazilian hospitals. The International Journal of Health Planning and Management, 32 (1), e99-e120. doi: https://doi.org/10.1002/hpm.2331.

Creswell, J. W. (2014). Research design: Qualitative, quantitative and mixed methods approaches. Newbury Park, CA: Sage Publications.

Crosby, L., Lortie, E., Rotenberg, B., \& Sowerby, L. (2019). Surgical Instrument Optimization to Reduce Instrument Processing and Operating Room Setup Time. Otolaryngology-Head and Neck Surgery, 1-5. doi: https://doi.org/10.1177/0194599819885635.

Curatolo, N., Lamouri, S., Huet, J. C., \& Rieutord, A. (2013). A Critical Analysis of Lean Approach in Hospital. Business Process Management Journal, 20(3), 433-454. doi: https://doi.org/10.1108/BPMJ-04-2013-0051.

D'Andreamatteo, A., lanni, L., Lega, F., \& Sargiacomo, M. (2015). Lean in healthcare: A comprehensive review. Health Policy, 119(9), 1197-1209. doi: https://doi.org/10.1016/j.healthpol.2015.02.002. 
Dobrzykowski, D. D., Mcfadden, K. L., \& Vonderembse, M. A. (2016). Examining pathways to safety and financial performance in hospitals: A study of lean in professional service operations. Journal of Operations Management, 42-43(1), 39-51. doi: https://doi.org/10.1016/j.jom.2016.03.001.

Drotz, E., \& Poksinska, B. (2014). Lean in healthcare from employees' perspectives. Journal of Health Organization and Management, 28(2), 177-195. doi: https://doi.org/10.1108/JHOM-03-20130066.

Farrelly, J. S., Clemons, C., Witkins, S., Hall, W., Christison-Lagay, E. R., Ozgediz, D. E., Cowles, R. A., Stitelman, D. H., \& Caty, M. G. (2017). Surgical tray optimization as a simple means to decrease perioperative costs. Journal of Surgical Research, 220, 320-326. doi: https://doi.org/10.1016/j.jss.2017.06.029.

Farrokhi, F. R., Gunther, M., Williams, B., \& Blackmore, C. C. (2015). Application of lean methodology for improved quality and efficiency in operating room instrument availability. Journal for Healthcare Quality. 37(5), 277-286. doi: https://doi.org/10.1111/jhq.12053.

Giraldo, A., Mothersell, W. M., \& Motwani, J. (2015). The need for implementing lean in healthcare organizations. International Journal Productivity and Quality Management, 16(2), 211-230. doi: https://doi.org/10.1504/IJPQM.2015.071240.

Graban, M. (2011). Lean hospitals: improving quality, patient safety, and employee satisfaction. New York: CRC Press.

Grosz, B. J. (1996). Collaborative Systems (AAAl-94 Presidential Address). Al magazine, 17(2), 67-85. doi: https://doi.org/10.1609/aimag.v17i2.1223

Hendry, C. (2012). Human resource management: a strategic approach to employment. London: Routledge.

Henrique, D. B., Rentes, A. F., \& Godinho, M., Fillho. (2016). A new value stream mapping approach for healthcare environments. Production Planning \& Control, 27(1), 24-48. doi: https://doi.org/10.1080/09537287.2015.1051159.

Hicks, C., McGovern, T., Prior, G., \& Smith, I. (2015). Applying lean principles to the design of healthcare facilities. International Journal of Production Economics, 170(Part B), 677-686. doi: https://doi.org/10.1016/j.ijpe.2015.05.029.

Holden, R. J., Eriksson, A., Andreasson, J., Williamsson, A., \& Dellve, L. (2015). Healthcare workers' perceptions of lean: A context-sensitive, mixed methods study in three Swedish hospitals. Applied Ergonomics, 47(1), p. 181-192. doi: https://doi.org/10.1016/j.apergo.2014.09.008.

Improta, G., Cesarelli, M., Montuori, P., Santillo, L. C., \& Triassi, M. (2017). Reducing the risk of healthcare-associated infections through Lean Six Sigma: The case of the medicine areas at the Federico II University Hospital in Naples (Italy). Journal of Evaluation in Clinical Practice, 24(2), 338-346. doi: https://doi.org/10.1111/jep.12844.

Jackson, T. L. (Ed.). (2012). Kaizen workshops for lean healthcare. CRC Press.

Johnson, J. E., Smith, A. L., \& Mastro, K. A. (2012). From Toyota to the bedside: nurses can lead the lean way in health care reform. Nursing administration quarterly, 36(3), 234-242. doi: https://doi.org/10.1097/ NAQ.0b013e318258c3d5. 
Joosten, T., Bongers, I., \& Janssen, R. (2009). Application of lean thinking to health care: issues and observations. International journal for quality in health care, 21(5), 341-347. doi: https://doi.org/10.1093/intqhc/mzp036.

Lindskog, P., Hemphälä, J., \& Eriksson, A. (2017). Lean tools promoting individual innovation in healthcare. Creativity and Innovation Management, 26(2), 175-188. doi: https://doi.org/10.1111/caim.12201.

Liu, X., Zhu, Y., Peng, K., Zhou, C., Liu, M., Liu, S. C., \& Tu, C. (2014). Application of PDCA Method in Quality Management of Running Medical Records at the Department of Orthopedics. Chinese Medical Record English Edition, 2(3), 112-115. doi: https://doi.org/10.3109/23256176.2014.910906.

Lunardini, D., Arington, R., Canacari, E. G., Gamboa, K., Wagner, K., \& Mcguire, K. J. (2014). Lean principles to optimize instrument utilization for spine surgery in an academic medical center: an opportunity to standardize, cut costs, and build a culture of improvement. Spine, 39(20), 1714-1717. doi: https://doi.org/10.1097/BRS.0000000000000480.

Machado, V. C., \& Leitner, U. (2010). Lean tools and lean transformation process in health care. International Journal of Management Science and Engineering Management, 5(5), 383-392. doi: https://doi.org/10.1080/17509653.2010.10671129.

Mannon, M. (2014). Lean healthcare and quality management: The experience of ThedaCare. Quality Management Journal, 21(1), 7-10. doi: https://doi.org/10.1080/10686967.2014.11918371

Marchand, K.B., Taylor, K.B., Salem, H.S., Mont, M.A., \& Marchand, R.C. (2020). Surgical Tray Optimization and Efficiency: The Impact of a Novel Sealed Sterile Container and Instrument Tray Technology. Surg Technol Int, Nov 27;37: sti37/1375. Advance online publication. PMID: 33245139.

Martins, R. A. (2012). Abordagens Quantitativa e Qualitativa. In P. A. C. Miguel (Org.). Metodologia de pesquisa em engenharia de produção e gestão de operações (pp. 47-63). Rio de Janeiro: Elsevier.

Ministério da Saúde. (2015). Despacho n. 1400-A/2015: Anexo - Plano Nacional para a Segurança dos Doentes 2015-2020. Diário da República, 2(28), 3882-(2)-3882-(10). Retrieved December 04, 2020, from https://dre.pt/application/file/66457154

Ministério da Saúde. (2016). Manual de boas práticas de gestão das Órteses, Próteses e Materiais Especiais (OPME). Retrieved November 05, 2020, from http://bvsms.saude.gov.br/bvs/publicacoes/manual_praticas_ gestao_proteses_materiais_especiais.pdf.

Montella, E., Di Cicco, M. V., Ferraro, A., Centobelli, P., Raiola, E., Triassi, M., \& Improta, G. (2016). The application of Lean Six Sigma methodology to reduce the risk of healthcare-associated infections in surgery departments. Journal of Evaluation in Clinical Practice, 23(3), 530-539. doi: https://doi.org/10.1111/jep.12662.

Moraros, J., Lemstra, M., \& Nwankwo, C. (2016). Lean interventions in healthcare: do they actually work? A systematic literature review. International Journal for Quality in Health Care, 28(2), 150-165. doi: https://doi.org/10.1093/intqhc/mzv123. 
Poksinska, B. B., Fialkowska-Filipek, M., \& Engström, J. (2016). Does Lean healthcare improve patient satisfaction? A mixed-method investigation into primary care. BMJ Quality \& Safety, 26(2), 8586. doi: https://doi.org/10.1136/bmjqs-2015-004290.

Rutledge, J., Xu, M., \& Simpson, J. (2015). Application of the Toyota Production System Improves Core Laboratory operation. American Journal of Clinical Pathology, 133(1), 24-31. doi: https://doi.org/10.1309/AJCPD1MSTIVZIOPZ.

Schonberger, R. J. (2018). Reconstituting lean in healthcare: From waste elimination toward 'queueless' patient-focused care. Business Horizons, 61(1), 13-22. doi: https://doi.org/10.1016/j.bushor.2017.09.001.

Sugianto, J. Z., Stewart, B., Ambruzs, J. M., Arista, A., Park, J. Y., Cope-Yokoyama, S., \& Luu, H. S. (2015). Applying the Principles of Lean Production to Gastrointestinal Biopsy Handling: From the Factory Floor to the Anatomic Pathology Laboratory. Laboratory Medicine, 46(3), 259-264. doi: https://doi.org/10.1309/LMJCUFUNOLT8C6DB.

Tay, H. L. (2016). Lean Improvement Practices: Lessons from Healthcare Service Delivery Chains. IFACPapersOnLine, 49(12), 1158-1163. doi: https://doi.org/10.1016/j.ifacol.2016.07.660.

Tay, H. L., Singh, P. J., Bhakoo, V., \& Al-Balushi, S. (2017). Contextual factors: assessing their influence on flow or resource efficiency orientations in healthcare lean projects. Operations Management Research, 10 (3-4), 118-136. doi: https://doi.org/10.1007/s12063-017-0126-3.

Tortorella, G., Dun, D. H., \& Almeida, A. G. (2020). Leadership behaviors during lean healthcare implementation: a review and longitudinal study. Journal of Manufacturing Technology Management, 31(1), 193-215. doi: https://doi.org/10.1108/JMTM-02-2019-0070.

Van Rossum, L., Aij, K. H., Simons, F. E., Eng, N. van der, \& Have, W. D. ten. (2016). Lean healthcare from a change management perspective: The role of leadership and workforce flexibility in an operating theatre. Journal of Health Organization and Management, 30(3), 475-493. doi: https://doi.org/10.1108/JHOM-06-2014-0090.

Waldhausen, J. H., Avansino, J. R., Libby, A., \& Sawin, R. S. (2010). Application of lean methods improves surgical clinic experience. Journal of pediatric surgery, 45(7), 1420-1425. doi: https://doi.org/10.1016/j.jpedsurg.2009.10.049

Wang, X. (2014). Application of PDCA Method in Medical Record Archiving Management. Chinese Medical Record English Edition, 2(1), 22-26. doi: https://doi.org/10.3109/23256176.2014.897487.

Wannemuehler, T. J., Elghouche, A. N., Kokoska, M. S., Deig, C. R., \& Matt, B. H. (2015). Impact of L ean on surgical instrument reduction: Less is more. The Laryngoscope, 125(12), 2810-2815. doi: https://doi.org/10.1002/lary.25407. Epub 2015 Jun 24.

Worth, J., Shuker, T., Keyte, B., Luckman, J., Ohaus, K., Verble, D., \& Nickel, T. (2012). Perfecting patient journeys. Lean Enterprise Institute.

Yin, R. K. (2015). Case study research: Design and Methods, Applied social research methods series. London: Sage Publications.

Zadeh, R. S., Shepley, M. M., \& Waggener, L. T. (2012). Rethinking efficiency in acute care nursing units: Analyzing nursing unit layouts for improved spatial flow. HERD: Health Environments Research \& Design Journal, 6(1), 39-65. doi: https://doi.org/10.1177/193758671200600103. 